\title{
Balanço cátion-aniônico da dieta no metabolismo de cálcio em ovinos
}

\author{
Dietary cation anion balance on calcium metabolism in sheep
Gustavo Ribeiro Del Claro ${ }^{1}$ Marcus Antonio Zanetti ${ }^{2}$ Lisia Bertonha Correa ${ }^{2}$ Arlindo Saran Netto ${ }^{2}$ Fernanda Alves de Paiva ${ }^{2}$ Marcia SaladineVieira Salles ${ }^{3}$

\section{RESUMO}

$O$ objetivo deste trabalho foi estudar o efeito do balanço cátion-aniônico da dieta (BCAD) no balanço e concentração sérica de cálcio em ovinos da raça Santa Inês. $O$ delineamento experimental foi em blocos para controlar o efeito do peso inicial dos animais. Foram utilizados cinco tratamentos com níveis crescentes de BCAD (-160, -40, 140, 250 e $500 \mathrm{mEq} / \mathrm{kg}$ de matéria seca) com cinco repetições em 25 animais. O BCAD influencia o metabolismo de cálcio em ovinos machos da raça Santa Inês. Com a diminuição do $B C A D$ o cálcio sérico aumentou. Dietas aniônicas aumentaram a excreção urinária de cálcio, entretanto a retenção não foi afetada.

Palavras-chave: ânion, $B C A D$, cátion, cálcio, $p H$

\section{ABSTRACT}

This work aimed at studing the effect of dietary cation-anionic balance $(D C A B)$ in calcium balance and serum concentration in Santa Ines breed sheep. To control the effect of animals' initial weight blocks were used as experimental design. Five treatments with increasing DCAB levels (-160, 40, 140, 250 and 500mEq/kg of dry matter) and five repetitions each were tested in 25 males. DCAB affected calcium metabolism of Santa Ines breed males. As DCAB lowered, serum calcium increased. Anionic diets raised urinary calcium excretion, however calcium retention wasn't affected.

Key words: anion, cation, calcium, DCAB, $p H$.

\section{INTRODUÇÃO}

O balanço cátion-aniônico dietético (BCAD), também conhecido por diferença cátion representa a diferença entre os cátions e os ânions fixos totais, presentes na dieta. Os ions $\mathrm{Na}, \mathrm{K}, \mathrm{Cl}$ e $\mathrm{SO}_{4}$ são escolhidos para o cálculo do BCAD por terem influência no balanço ácido-base, entretanto, podem alterar outras funções fisiológicas como o balanço osmótico, mecanismos de transporte de membrana e os impulsos nervosos (BLOCK, 1994). A influência do BCAD sobre o $\mathrm{pH}$ sangüíneo ocorre primeiramente no trato gastrintestinal, uma vez que a absorção de cátions é acompanhada da secreção de $\mathrm{H}^{+}$e a de ânions acompanhada da absorção de $\mathrm{H}^{+}$ou secreção de $\mathrm{HCO}_{3}^{-}$ (BLOCK, 1994). Outra forma de alteração diz respeito aos mecanismos de reabsorção promovidos pelos rins. A combinação desses mecanismos pode induzir a um estado de alcalose ou acidose, alterando o desempenho animal e também o metabolismo de macrominerais.

Nos últimos anos, muito se tem pesquisado sobre a manipulação do equilíbrio ácido-básico na prevenção de doenças metabólicas. Os estudos, na produção animal, iniciaram-se na avicultura de corte, entretanto, é na bovinocultura leiteira que as pesquisas se concentram, principalmente, na prevenção de distúrbios como a hipocalcemia peri-parto.

Em se tratando de BCAD e metabolismo de cálcio, existem algumas controvérsias literárias que merecem ser esclarecidas. O aumento do cálcio sangüíneo, oriundo de uma diminuição do BCAD pode ocorrer em função de alterações na absorção intestinal do cálcio, na função renal, ou por maior mobilização óssea. LOMBA et al. (1978) demonstraram um aumento da absorção de cálcio com uso de dietas aniônicas.

${ }^{1}$ Faculdade de Zootecnia e Engenharia de Alimentos, (FZEA), Universidade de São Paulo, (USP), rua Duque de Caxias Norte, 225 13630, Pirassununga, SP, Brasil. E-mail: gdelclaro@yahoo.com.br

${ }^{2}$ FZEA, USP, Pirassununga, SP, Brasil

${ }^{3}$ Instituto de Zootecnia Assis, Assis, SP, Brasil 
Entretanto, GOMIDE et al. (2004), em estudo desenvolvido com ovinos, encontraram decréscimo da absorção e retenção de cálcio com aumento da excreção fecal no aumento dos níveis de BCAD. Com relação à alteração do BCAD negativo na função renal, TAKAGI \& BLOCK (1991), utilizando-se de ovinos, demonstraram que dietas acidogênicas aumentam a excreção urinária de cálcio, entretanto DELAQUIS \& BLOCK (1995), em experimento com vacas em lactação com tratamentos de 258,1 e 55,5mEq/kg de MS, não encontraram aumento na concentração urinária de cálcio com a diminuição do BCAD. GOMIDE et al. (2004), em experimento conduzidos com ovinos machos e tratamento de $-12 \mathrm{mEq} / \mathrm{kg}$, também não encontraram aumento da excreção urinária de cálcio em relação aos tratamentos com BCAD positivo.

Além dos mecanismos indiretos do aumento da mobilização óssea em função de um BCAD negativo, alguns autores citam mecanismos específicos. A reabsorção óssea é mediada pelo paratormônio (PTH) e 1,25(OH) ${ }_{2} \mathrm{D}_{3}$. BLOCK (1984) mostrou aumento da mobilização óssea, coincidindo com o aumento de PTH e 1,25(OH) ${ }_{2} \mathrm{D}_{3}$. A reabsorção óssea de cálcio exige, pelo menos, 48 horas de estimulação pelo PTH. Para BYERS (1994), uma alimentação com dieta aniônica deveria ser utilizada, entre três a cinco semanas antes do parto. DEL CLARO et al. (2002) observaram que bovinos em crescimento submetidos a um BCAD de $-150 \mathrm{mEq} / \mathrm{kg}$ de MS, tiveram aumentos dos níveis séricos de cálcio total, 19 dias após o início da suplementação com sais aniônicos.

O NRC (1985) recomenda o uso de $0,5 \%$ de sulfato de amônia ou cloreto de amônia na ração total, com o objetivo da diminuir a urolitíase em ovinos. Essa prática poderia diminuir de forma indireta o BCAD modificando o metabolismo de cálcio. O uso de BCAD positivo foi reportado por FAUCHON et. al., (1995) para melhora do desempenho de ovinos. Entretanto, a relação do BCAD com o metabolismo de cálcio, nesse caso, foi pouco estudada.

O presente trabalho teve por objetivo verificar os efeitos de diferentes relações entre cátions e anions sobre o metabolismo de cálcio através do estudo da absorção aparente, da retenção e da concentração sérica desse macromineral em ovinos da raça Santa Inês.

\section{MATERIAL E MÉTODOS}

O estudo foi conduzido na Faculdade de Zootecnia e Engenharia de Alimentos da Universidade de São Paulo, no Campus de Pirassununga. Foram utilizados 25 cordeiros machos da raça Santa Inês com peso médio de $26,5 \mathrm{~kg}$ e idade média de 150 dias. Os animais foram alocados em gaiolas de estudo de metabolismo, equipadas com bebedouro automático, cocho e coletor de urina. Os ovinos foram alimentados com dieta calculada para suprir suas necessidades, segundo o NRC (1985). Foram oferecidos cinco tratamentos, diferindo nas concentrações de bicarbonato de sódio, sulfato de amônio e caulim em 5 níveis de BCAD $(-160 ;-40 ; 140 ; 260 ; 500 \mathrm{mEq} / \mathrm{kg}$ de MS). O tratamento com $140 \mathrm{mEq} / \mathrm{kg}$ de MS foi considerado o controle, uma vez que continha os níveis de $\mathrm{Na}^{+} ; \mathrm{K}^{++}$; $\mathrm{Cl}^{-}$e $\mathrm{S}^{-2}$ preconizados pelo NRC (1985) para ovinos. A tabela 1 apresenta a composição químico-bromatológicas das dietas experimentais.

O experimento teve duração de 90 dias, sendo os 15 primeiros correspondentes ao período de adaptação à ração total. Para a realização do balanço mineral, foram coletadas amostras de fezes e da dieta na proporção de $10 \%$. A urina foi amostrada na proporção de $5 \%$. O período de coleta teve duração de cinco dias, e foi realizado do $25^{\circ}$ ao $30^{\circ}$ dia e posteriormente entre o $55^{\circ}$ e o $60^{\circ}$ dia.

As determinações de matéria seca e matéria mineral para fezes e rações obedeceram às recomendações da AOAC (1990). O cálcio foi determinado por espectrofotometria de absorção atômica (equipamento Perkin Elmer), através de diluições e adição de $1 \%$ de Lantânio $(10 \mathrm{~N})$. O sódio foi determinado através de fotômetro de chama. $\mathrm{O}$ enxofre foi determinado por turbidimetria em sistema 'Flow Injection Analysis". O cloro foi determinado através de titulometria com nitrato de prata. As urinas, armazenadas individualmente, foram homogeneizadas, alocadas em garrafas plásticas e posteriormente acidificadas até atingirem $\mathrm{pH}$ entre 1 e 2. As análises de cálcio e sódio seguiram as metodologias apresentadas anteriormente para as rações e fezes. Os níveis de cálcio total e ionizado no soro foram analisados através de "Kit's" da marca LABORLAB. Para a análise do cálcio ionizado, foram utilizados 3 tipos de Kit's. O primeiro foi utulizado para a determinação da quantidade de albumina presente no soro; o segundo, para a determinação da proteína total, e o terceiro, para a determinação do cálcio total. Esses valores foram equacionados para a determinação do cálcio ionizado.

O delineamento experimental foi em blocos, para controlar o efeito do peso inicial dos animais, sendo que foram utilizados 25 animais com cinco repetições por tratamento, cinco tratamentos e cinco blocos. Os dados obtidos foram analisados por meio do procedimento GLM do programa computacional SAS (SAS Institute Inc., 1988), sendo realizada análise 
Tabela 1 - Composição químico-bromatológica e percentual dos minerais nas dietas experimentais com base na matéria seca e balanço cátion aniônico da dieta (BCAD) em mEq de $\left\{(\mathrm{Na}+\mathrm{K})-\left(\mathrm{Cl}+\mathrm{SO}_{4}\right)\right\}$.

\begin{tabular}{llllll}
\hline & -160 & -40 & 140 & 250 & 500 \\
\hline Proteína bruta (\%) & 19,15 & 20,47 & 18,63 & 20,98 & 19,37 \\
Fibra bruta (\%) & 9,46 & 8,56 & 8,63 & 8,16 & 9,11 \\
Extrato etéreo (\%) & 7,24 & 6,70 & 6,38 & 6,70 & 6,70 \\
Matéria mineral (\%) & 8,52 & 9,10 & 10,99 & 9,73 & 9,64 \\
Cálcio (\%) & 1,11 & 1,11 & 1,10 & 1,12 & 0,13 \\
Fósforo (\%) & 0,55 & 0,55 & 0,55 & 0,55 & 0,55 \\
Sódio (\%) & 0,35 & 0,35 & 0,35 & 0,65 & 1,13 \\
Potássio (\%) & 1,25 & 1,25 & 1,28 & 0,37 & 0,36 \\
Enxofre (\%) & 0,80 & 0,60 & 0,35 & 0,40 & 0,40 \\
Cloro (\%) & 0,46 & 0,43 & 0,44 & 251,01 & 500,49 \\
BCAD & $-159,88$ & $-40,54$ & 138,79 & & \\
\hline
\end{tabular}

de regressão (linear quadrática e cúbica) nos níveis do BCAD (-160, -40, 140, 250 e 500mEq/kg de MS). Foram realizados contrastes ortogonais entre os tratamentos $\mathrm{N} 1(-160 \mathrm{mEq} / \mathrm{kg}$ de MS), N2 (-40mEq/kg de MS), C (140mEq/kg de MS), P1 (+250mEq/kg de MS) e P2 (+500 $\mathrm{mEq} / \mathrm{kg}$ de $\mathrm{MS})$, e também entre os tratamentos negativos (N1 e N2), o controle (C) e os positivos (P1 e $\mathrm{P} 2$ ), foi considerado o nível de significância de $5 \%$ para todos os dados analisados. Os contrastes foram usados para uma comparação mais ampla entre os tratamentos de forma a esclarecer o efeito do BCAD nas variáveis usadas.

\section{RESULTADOS E DISCUSSÃO}

Os resultados dos balanços de cálcio estão apresentados nas tabelas 2 e 3 . Pela tabela 2, observase que o único efeito foi na excreção urinária de cálcio, a qual aumentou de forma linear com a diminuição do BCAD. A tabela 3 revela que, após cinqüenta e cinco dias de oferecimento das dietas iônicas, o comportamento do balanço de cálcio se alterou. A absorção aparente de cálcio apresentou efeito cúbico significativo, sendo que o mesmo comportamento foi observado para a retenção desse macromineral. A excreção urinária de cálcio apresentou comportamento quadrático, e foi sempre maior para os tratamentos negativos (N1 e N2).

Existe muita controvérsia na literatura, quanto aos efeitos do BCAD na absorção de cálcio. $\mathrm{O}$ presente experimento não encontrou efeito do BCAD na absorção de cálcio no primeiro balanço, porém encontrou efeito cúbico significativo no segundo balanço, o que pode ter acontecido por uma mudança no metabolismo de cálcio em função do tempo de oferecimento das dietas iônicas.

LECLERC \& BLOCK (1989) e DEL CLARO et al. (2002) não encontraram alterações na absorção intestinal com uso de dietas aniônicas ou catiônicas em ruminantes. SCHONEWILL et al. (1994) encontraram maior absorção aparente de cálcio com dietas aniônicas. LOMBA et al. (1978) afirmaram que a maior absorção se dá pela natureza acidogênica dos ânions no intestino, que aumenta a solubilização do cálcio. Para BLOCK (1994), não existe um mecanismo claro de influência do BCAD na absorção intestinal de cálcio, o que explica a divergência de resultados na literatura. Em geral, os resultados encontrados no primeiro balanço do presente experimento têm uma maior prevalência na literatura, o que pode acontecer pelo tempo de oferecimento das dietas iônicas, uma vez que a maior parte dos experimentos são feitos com vacas no préparto e oferecimento de dietas aniônicas por cerca de um mês.

Segundo MACkDOWELL (1992), as fezes são a principal via de excreção do cálcio, sendo o cálcio das fezes resultado do cálcio não absorvido da dieta, cálcio endógeno não absorvido e da secreção intestinal desse elemento. O efeito cúbico encontrado no segundo balanço pode ter sido em função de uma secreção intestinal de cálcio, o que dificulta a interpretação desses resultados.

Quanto à excreção urinária, no primeiro balanço, foi encontrado efeito linear significativo. No 
Tabela 2 - Balanço de cálcio entre o $25^{\circ}$ e $30^{\circ}$ dia em ovinos, alimentados com diferentes níveis de balanço cátion aniônico da dieta (BCAD) em $\mathrm{mEq}$ de $\left\{(\mathrm{Na}+\mathrm{K})-\left(\mathrm{Cl}+\mathrm{SO}_{4}\right)\right\}$.

\begin{tabular}{|c|c|c|c|c|c|c|c|c|c|}
\hline \multirow[t]{2}{*}{ Variáveis } & \multicolumn{6}{|c|}{ Tratamentos } & \multicolumn{3}{|c|}{ Nível de significância } \\
\hline & -160 & -40 & 140 & 250 & 500 & $\mathrm{CV} \%$ & $\mathrm{~L}$ & $\mathrm{Q}$ & $\mathrm{C}$ \\
\hline Consumo & 0,846 & 0,882 & 0,829 & 0,878 & 0,891 & - & - & - & - \\
\hline Fezes $^{1}$ & 0,291 & 0,267 & 0,388 & 0,358 & 0,308 & 15,16 & 0,10 & 0,01 & 0,08 \\
\hline Fezes $(\%)^{*}$ & 34,288 & 30,347 & 46,953 & 40,887 & 34,578 & 13,63 & 0,14 & 0,01 & 0,04 \\
\hline Urina $^{1}$ & 0,003 & 0,004 & $<0,001$ & $<0,001$ & $<0,001$ & 60,63 & $<0,01$ & $<0,01$ & 0,11 \\
\hline Urina $(\%)^{*}$ & 0,415 & 0,399 & 0,092 & 0,071 & 0,084 & 63,86 & $<0,01$ & 0,03 & 0,17 \\
\hline Abs. Aparente ${ }^{1}$ & 0,555 & 0,614 & 0,441 & 0,520 & 0,582 & 7,63 & 0,45 & $<0,01$ & $<0,01$ \\
\hline Abs. Aparente $(\%)^{*}$ & 65,711 & 69,652 & 54,571 & 59,112 & 65,421 & 8,40 & 0,16 & $<0,01$ & 0,04 \\
\hline Retenção $^{1}$ & 0,551 & 0,610 & 0,440 & 0,519 & 0,582 & 7,62 & 0,53 & $<0,01$ & 0,01 \\
\hline Retenção $(\%)^{*}$ & 65,297 & 69,252 & 52,954 & 59,040 & 65,337 & 8,20 & 0,17 & $<0,01$ & $<0,04$ \\
\hline
\end{tabular}

${ }^{1}$ gramas por $\mathrm{kg}{ }^{0,75} / \mathrm{dia} .{ }^{*}$ Calculados em relação ao consumido.

CV Coeficiente de variação.

L - Efeito Linear Q- Efeito Quadrático C- Efeito Cúbico

Tabela 3 - Balanço de cálcio entre o $55^{\circ}$ e $60^{\circ}$ dia de em ovinos, alimentados com diferentes níveis de balanço cátion aniônico da dieta (BCAD) em mEq de $\left\{(\mathrm{Na}+\mathrm{K})-\left(\mathrm{Cl}+\mathrm{SO}_{4}\right)\right\}$.

\begin{tabular}{|c|c|c|c|c|c|c|c|c|c|}
\hline \multirow[t]{2}{*}{ Variáveis } & \multicolumn{5}{|c|}{ Tratamentos. } & \multicolumn{4}{|c|}{ Nível de significância } \\
\hline & -160 & -40 & 140 & 250 & 500 & $\mathrm{CV} \%$ & $\mathrm{~L}$ & Q & $\mathrm{C}$ \\
\hline Consumo & 0,798 & 0,830 & 0,783 & 0,810 & 0,814 & - & - & - & - \\
\hline Fezes $^{1}$ & 0,385 & 0,348 & 0,388 & 0,305 & 0,384 & 16,01 & 0,82 & 0,15 & 0,50 \\
\hline Fezes $(\%)^{*}$ & 48,22 & 41,96 & 50,93 & 37,68 & 47,05 & 13,87 & 0,74 & 0,27 & 0,61 \\
\hline Urina $^{1}$ & 0,002 & 0,003 & $<0,001$ & 0,002 & 0,001 & 60,61 & 0,05 & 0,42 & 0,30 \\
\hline Urina $(\%)^{*}$ & 0,324 & 0,414 & 0,128 & 0,237 & 0,189 & 61,29 & 0,05 & 0,42 & 0,40 \\
\hline Abs. Aparente ${ }^{1}$ & 0,413 & 0,482 & 0,377 & 0,504 & 0,428 & 11,01 & 0,77 & 0,37 & 0,88 \\
\hline Abs. Aparente $(\%)^{*}$ & 51,770 & 58,032 & 49,065 & 61,317 & 52,943 & 11,49 & 0,74 & 0,27 & 0,61 \\
\hline Retenção $^{1}$ & 0,410 & 0,478 & 0,376 & 0,503 & 0,427 & 11,10 & 0,73 & 0,36 & 0,86 \\
\hline Retenção $(\%)^{*}$ & 51,450 & 57,620 & 48,936 & 62,079 & 52,753 & 11,57 & 0,71 & 0,26 & 0,59 \\
\hline
\end{tabular}

${ }^{1}$ gramas por kg ${ }^{0,75} /$ dia. * Calculados em relação ao consumido.

CV ${ }^{1}$ Coeficiente de variação.

L - Efeito Linear Q- Efeito Quadrático C- Efeito Cúbico

segundo balanço experimental, o efeito observado foi quadrático. Nos dois balanços, foram encontrados aumento da excreção urinária de cálcio com a queda do $\mathrm{BCAD}$. Os resultados concordam com os relatados por VAGNONI e OETZEL (1998), SETTI (2001) e DEL
CLARO et al. (2002). SCHONEWILLIE et al. (1999) afirmaram que a excreção urinária de cálcio foi seis vezes maior nas vacas com tratamento aniônico, do que naquelas com tratamento catiônico. VAN MOSEL et al. (1993) afirmaram que vacas alimentadas com dieta 
aniônica excretam de 10 a 30 vezes mais cálcio que vacas alimentadas com dieta catiônica. DELAQUIS \& BLOCK (1995) não encontraram diferenças na excreção urinária de cálcio com a diminuição do BCAD provavelmente em função da pouca diferença entre os dois tratamentos (258,1 e 55,5mEq/kg de MS). GOMIDE et al. (2004), em trabalho com ovinos, também não encontraram aumento de excreção de cálcio na urina com uso de dieta aniônica, provavelmente por ela ser pouco negativa $(-12 \mathrm{mEq} / \mathrm{kg}$ de $\mathrm{MS})$.

O aumento da excreção urinária, segundo BLOCK (1984), é uma conseqüência secundária do aumento sanguíneo em função da mobilização óssea. Com esse aumento da excreção de cálcio, ocorreria um aumento da formação de $1,25(\mathrm{OH})$ que estimularia a mobilização óssea e aumento sanguíneo de cálcio. Outro mecanismo seria em função da produção renal de $1,25(\mathrm{OH})_{2} \mathrm{D}_{3}$, a qual poderia ser influenciada pelo baixo pH intracelular (BLOCK,1994).

A excreção de cálcio da dieta é predominantemente feita pelas fezes. Dessa forma, a alteração da excreção urinária do cálcio não foi suficiente para influenciar a retenção desse macromineral. Não houve influência do BCAD na retenção de cálcio no primeiro balanço e foram encontrados efeitos cúbicos significativos do BCAD no segundo balanço, provavelmente em função da secreção intestinal de cálcio.

Os resultados apresentados concordam com os encontrados por TAKAGI \& BLOCK (1991) os quais relataram absorção aparente similar entre os tratamentos e aumento de excreção urinária não suficiente para alterar a retenção de cálcio. FREDEEN et al. (1988), em experimento conduzido com vacas no pré-parto, não observaram alteração na excreção fecal de cálcio em função do decréscimo do BCAD. No entanto, diferenças no balanço de cálcio não foram relatadas pelos autores, tanto para as vacas em produção, como para as em pré-parto. Para as vacas em pré-parto, a excreção urinária de cálcio aumentou de 0,59 para $7,6 \%$ do total de cálcio consumido. WILSON et al. (1998), trabalhando com cordeiros obtiveram um acréscimo no cálcio excretado de $0,11 \mathrm{~g} /$ dia na dieta controle para $0,75 \mathrm{~g} /$ dia na dieta aniônica. A absorção aparente desse mineral não se alterou, ou seja, o decréscimo no BCAD foi suficiente para alterar a excreção urinária, sem afetar o balanço de cálcio, assim como no presente experimento.

Dessa forma, o BCAD parece não ter influência direta sobre a absorção intestinal de cálcio, contudo tem efeito sobre a excreção urinária, a qual aumenta com o decréscimo do BCAD. Esse efeito, apesar de efetivo, não é suficiente para influenciar a retenção de cálcio.
A tabela 4 apresenta os níveis do cálcio total e ionizado ( $\mathrm{mg}$ por $\mathrm{dL}$ ) durante o experimento. Observase que, para o cálcio total, no $15^{\circ}$ e $60^{\circ}$ dia, houve um efeito linear significativo, ou seja, com o decréscimo do BCAD, houve um aumento dos níveis séricos de cálcio total. No $30^{\circ}$ dia, apesar do efeito cúbico, os tratamentos negativos apresentaram maiores concentrações de cálcio que os positivos e o controle. De maneira geral, tanto na forma ionizada como na total, a concentração de cálcio foi superior nos tratamentos N1 e N2. Para o cálcio ionizado, a análise de contrastes revelou que as dietas negativas (N1 e N2) foram superiores às positivas ( $\mathrm{P} 1 \mathrm{e} \mathrm{P} 2) \mathrm{e}$ a controle.

Do cálcio total $(\mathrm{mg} / \mathrm{dL})$ encontrado no sangue, 45 a $50 \%$ estão na forma solúvel, ou seja, ionizada. Cerca de 40 a 45\% estão ligados à proteína, os outros $5 \%$ formam complexos com elementos inorgânicos de acordo com o pH do sangue (CUNNINGHAM ,1999). Os maiores níveis encontrados nos tratamentos com inclusão de sal aniônico em ovinos machos concordam com os relatados por TAKAGI \& BLOCK (1991).

BLOCK (1984), trabalhando com níveis de $+330,5$ e -128,5mEq por kg de MS, observou uma maior concentração plasmática de cálcio no BCAD negativo, segundo o autor resultado de um aumento na mobilização óssea. LECLERC \& BLOCK (1989) concluíram que a alimentação com BCAD negativo durante o pré-parto reduz o declínio natural da concentração de Ca. Esse resultado, segundo os autores, ocorre de efeito indireto da acidose metabólica, afetando a reabsorção renal de cálcio e a mobilização óssea desse macromineral.

DEL CLARO et al. (2002) observaram aumento no nível de cálcio total no soro 19 dias após o oferecimento de dieta aniônica $(-150 \mathrm{mEq} / \mathrm{kg})$. VAGNONI \& OETZEL (1998) trabalharam com vacas secas e BCAD de $203 ;-51 ;-40$ e $-63 \mathrm{mEq}(\mathrm{Na}+\mathrm{K})-(\mathrm{Cl}+\mathrm{S}) / \mathrm{kg}$ de $\mathrm{MS}$ e não encontraram alterações nos níveis de cálcio total e ionizado entre os períodos em que as vacas foram submetidas ao tratamento de $203 \mathrm{mEq} / \mathrm{kg}$ de MS em relação aos tratamentos com sais aniônicos. A adição de bicarbonato de sódio na ração de vacas não promoveu nenhuma variação na concentração sérica do cálcio (TUCKER et al., 1991). FAUCHON et al. (1995), em experimento com ovinos em crescimento, com tratamentos de 4, 175, 390 e $580 \mathrm{mEq}(\mathrm{Na}+\mathrm{K})-(\mathrm{Cl}+\mathrm{S})$ / $\mathrm{kg}$ de MS não encontraram diferenças na concentração de cálcio plasmático, entretanto os autores afirmaram que os animais desse experimento não estavam sob condições de acidose, a qual iria propiciar a reabsorção óssea. GOFF et al. (1991) mostraram que a redução do BCAD aumentou a produção de $1,25(\mathrm{OH})_{2} \mathrm{D}_{3}$ por unidade de PTH. Então, a acidose metabólica 
Tabela 4 - Níveis de cálcio sérico total e ionizado $(\mathrm{mg} / \mathrm{dL})$ em ovinos alimentados com diferentes níveis de balanço cátion aniônico da dieta (BCAD) em mEq de $\left\{(\mathrm{Na}+\mathrm{K})-\left(\mathrm{Cl}+\mathrm{SO}_{4}\right)\right\}$.

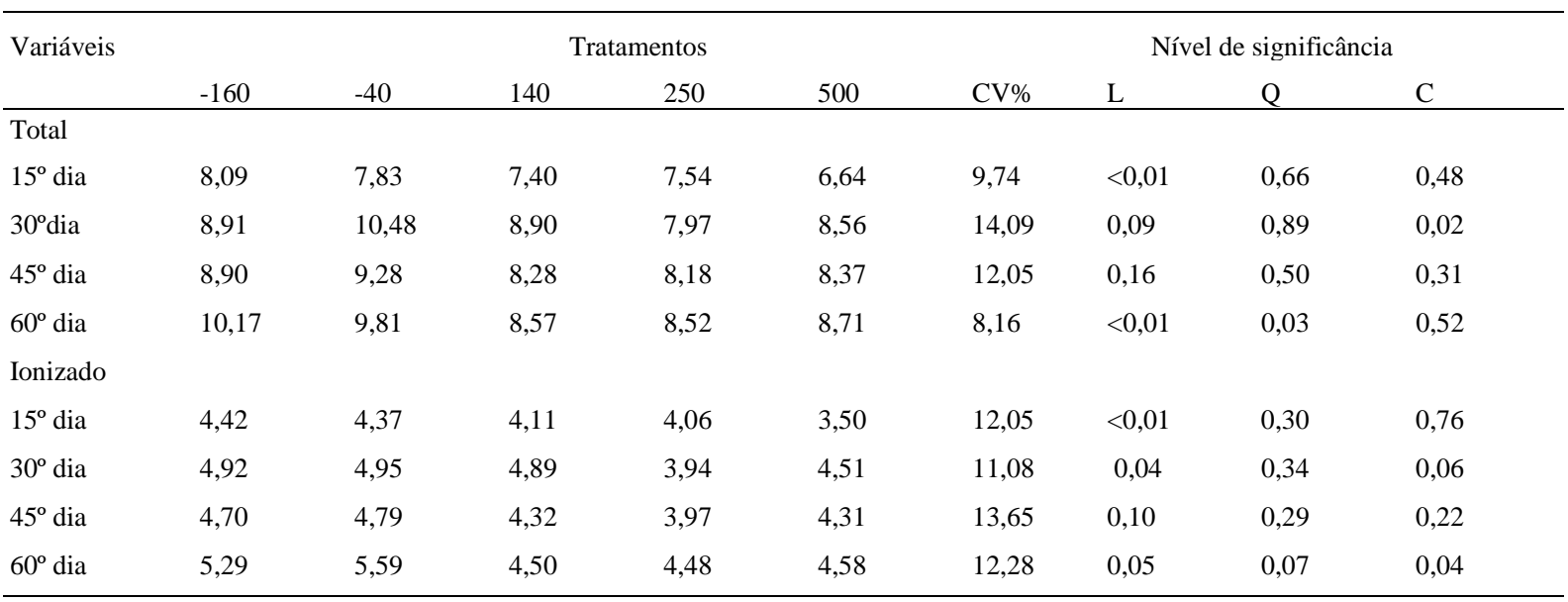

CV-Coeficiente de variação.

L - Efeito Linear Q- Efeito Quadrático C- Efeito Cúbico

proporcionada pelo BCAD negativo estimula a transformação de $25(\mathrm{OH})$ colicalciferol em $1,25(\mathrm{OH})_{2} \mathrm{D}_{3}$, a qual aumenta a reabsorção óssea liberando cálcio para a corrente sangüínea, o que pode explicar em parte, os resultados do presente estudo.

\section{CONCLUSÕES}

O BCAD influencia o metabolismo de cálcio em ovinos machos. A redução do BCAD aumenta a excreção urinária de cálcio, entretanto, não altera a retenção desse macromineral. A concentração sanguínea de cálcio aumenta com a diminuição do BCAD 15 dias após o início da suplementação com sulfato de amônia.

\section{REFERÊNCIAS}

ASSOCIATION OF OFFICIAL ANALYTICAL CHEMISTS. Official methods of analysis. 15.ed. Arlington: AOAC, 1990. 1298p.

BLOCK, E. Manipulating dietary anions and cations for prepartum dairy cows to reduce incidence of milk fever. Journal of Dairy Science, Champaingn, v.67, n.12, p.2939$2948,1984$.

BLOCK, E. Manipulation of dietary cation-anion difference on nutritionally reted production diseases, productivity, and metabolic responses of dairy cows. Journal of Dairy Science, Champaingn, v.77, n.5, p.1437-1450, 1994.
BYERS, D.I. Management considerations for successful use of anionic salts in dry-cow diets. Compendim on Continuing education for the practicing Veterinarian, v.16, n.1-3, p.237-242, 1994.

CUNNINGHAM, J.G. Tratado de fisiologia veterinária. Rio de Janeiro: Guanabara, 1999. 454p.

DEL CLARO, G.R. et al. Influência da dieta aniônica no balanço macromineral em novilhos holandeses. Arquivo Brasileiro de Medicina Veterinária e Zootecnia, v.74, n.3, p.281283, 2002

DELAQUIS, A.M.; BLOCK, E. Acid-base satatus, renal function, water, and macromineral metabolism of dry cows. Journal of Dairy Science, Champaingn, v.78, n.4, p.604619, 1995.

FAUCHON, C. et al. Effects of dietary cation-anion concentrations on performance and acid-base balance in growing lambs. Canadian Journal of Animal Science, v.75, p.145$151,1995$.

FREDEEN, A et al. Characterization of acid base disturbances and effects on calcium and phosphorus balances of dietary fixed ions in pregnant or lactating does. Journal of Animal Science, Champaign, v.66, p.159-173, 1988.

GOFF, J.P. et al. Addition of choride to a prepartal diet hight in cations increases 1,25-dihydroxyvitamin $d$ response to hypocalcemia preventing milk fever. Journal of Dairy Science, Champaingn, v.74, n.11, p.3863-3871, 1991.

GOMIDE, C.A. et al. Influência da diferença cátion-aniônica da dieta sobre o balanço de cálcio, fóforo e magnésio em ovinos. Arquivo Brasileiro de Medicina veterninária e Zootecnia, v.56, n.3, p.363-369, 2004.

LECLERC, H.; BLOCK, E. Effects of reducing dietary cationanion balance prepartum dairy cows with specific refernce to 
hypocalm parturient paresis. Canadian Journal of Animal Science, v.69, p.411-423, 1989.

LOMBA, F. et al. Calcium digestibiliy in cows as influenced by ecess of alkaline ions over stable acid in their diets. British Journal of Nutrition, v.39, p.425-429, 1978.

McDOWELL, L.R. Minerals in animal and human nutrition. New York: Academic, 1992. 232p.

NATIONAL RESEARCH COUNCIL. Nutrient requeriment of sheep. Washington, D.C.: National Academy of Sciences, 1985. 99p.

SAS Institute Inc. SAS stat guide. Release 6.03 Edition. Cary, NC, 1988. 1028p.

SCHONEWILLE, J.T. et al. Stimulatory effect of anion (chloride) rich ration on apparent calcium absorption in dairy cows. Livestock Production Science, v.40, p.233-240, 1994.

SCHONEWILLE, J.T. et al. Hipocalcemia induced by intravenous Administration of disodium ethylenediaminotetraacetate and its effects on excretion of calcium in urine of cows. Journal of Dairy Science, v.82 p.1317-1324,1999.

SETTI, M.C. Parâmetros metabólicos e balanço cátionaniônico da dieta (BCAD) para vacas da raça Holandesa.
2001. 209f. Tese (Doutorado em Produção animal) - Faculdade de Ciências Agrárias e Veterinárias, UNESP.

TAKAGI, H.; BLOCK, E. Effects of manipulating dietary cation-anion balance on macromineral balance in sheep. Journal of Dairy Science, Champaingn, v.74, n.12, p.42024214, 1991.

TUCKER, W.B. et al. Role of sulfur and chloride in dietary cation-anion balance equation for lactating dairy cattle Journal of Dairy Science, Champaingn, v.69, n.3, p.12051213, 1991.

VAGNONI, D.B.; OETZEL, G.R. Effects of dietary cationanion differnce on the acid-base status of dry cows. Journal of Dairy Science, Champaingn, v.81, p.1643-1652, 1998.

VAN MOSEL, M. et al. Effectts of reducing dietary cationanion balance on rate of calcium mobilization by dairy cows at parturition. Research in Veterinary Science, v.54, p.1-9, 1993.

WILSON, K.L et al. The effects of a cation-anion balanced diet on calcium and phosphorus metabolism in growing lambs. Proceedings of the New Zealand Society of Animal Production, v.58, p.192-194, 1998. 